\title{
Developmental Changes in Myocardial Contractile Reserve in the Lamb
}

\author{
DAVID F. TEITEL, DANIEL SIDI, ${ }^{1}$ TOM CHIN, CLAIRE BRETT, MICHAEL A. HEYMANN, AND \\ ABRAHAM M. RUDOLPH \\ Cardiovascular Research Institute and the Departments of Pediatrics, Physiology, and Obstetrics, Gynecology \\ and Reproductive Sciences, University of California, San Francisco, California 94143
}

\begin{abstract}
We have assessed serial changes in myocardial contractility and reserve in the normal lamb over the first month of life using an in vivo adaptation of the endsystolic pressure-volume relationship. Via a left thoracotomy, we insert a catheter tip pressure transducer into the left ventricle, affix an echo transducer onto the left ventricular epicardium, place an electromagnetic flow transducer around the pulmonary artery, and insert catheters for monitoring and infusions. We measure contractility by generating left ventricular wall stress-volume index (the cube of dimension) curves, at the same time increasing afterload by infusing phenylephrine. The slope of the endsystolic wall stress-volume index relationship is our index of contractility. Weekly studies were performed at rest and during isoproterenol infusion in 12 animals, and after propranolol administration in four. The data showed a progressive decrease in resting contractility but no change in maximal contractility during isoproterenol infusion over the 4 wk. Taking each week separately, the average increase in contractility during isoproterenol infusion was small at 1 wk (13\%), moderate at 2 and 3 wk ( 24 and $26 \%$, respectively), and large at 4 wk ( $79 \%)$. $\beta$-Adrenergic blockade with propranolol caused a significant decrease in contractility in three of four animals studied at $1 \mathrm{wk}$, in only one of four animals at $2 \mathrm{wk}$, and in none of four animals at 3 or 4 wk. Thus, the newborn lamb shows a limited reserve in contractility that increases progressively with age; the limited reserve appears secondary to a high resting $\beta$ adrenergic state. (Pediatr Res 19: 948-955, 1985)
\end{abstract}

\section{Abbreviation}

Emax, maximal ventricular elastance

Studies of left ventricular performance have shown that the newborn lamb has a limited ability to increase cardiac output. Klopfenstein and Rudolph (1) reported a lesser response to volume loading in newborn as compared to older lambs (4 and 6 wk of age). This was substantiated by Romero and Friedman

Received February 13, 1984: accepted April 10, 1985

Address for correspondence and reprint requests David F. Teitel, Room 1315 M, University of California at San Francisco, San Francisco, CA 94143.

D.F.T. was supported by a research fellowship from the Medical Research Council of Canada during the initial phases of this project and is currently supported by an NIH New Investigator Award (HL 30457). This project was supported in part by an NIH Research Grant (HL 23681) and an NIH Program Project Grant (HL 24056).

1 Present address: Service de Cardiologie Infantile, Hôpital Necker-Enfants Malades, Rue Sevrès, Paris 75015. France.
(2) who showed a decrease in stroke volume during volume loading that they ascribed to a mismatch between the increase in afterload and the ability to increase inotropic state. The response to digoxin was similarly blunted in the newborn as compared to the adult sheep (3). Studies of fetal myocardium have shown immaturity of structure, function, and sympathetic innervation relative to the adult $(4,5)$. These developmental differences may limit the response of the newborn heart. High resting indices of myocardial performance, however, have been seen in the newborn as compared to the adult sheep $(6,7)$. Thus the newborn may be unable to substantially improve ventricular performance because, even though the myocardium is fully or almost fully developed, it may already be functioning at maximal contractility because of high demand. The presence or absence of a reserve in contractility, whatever the cause, would have major importance when inotropic agents are used to treat heart failure in the human newborn. We therefore devised a protocol to assess directly contractility and contractile reserve in the newborn.

Descriptors, or indices, of the ability of the heart to eject blood (pump function) may depend on one or several of the determinants of ventricular performance. The four major determinants of performance are heart rate, contractility, preload, and afterload. For any such index to describe only the intrinsic contractility (muscle function) of the heart, it must be independent of heart rate and the loading conditions of the heart (8-11). Many attempts to use single-measurement indices to assess contractility have failed because these indices are dependent on the other determinants, and overall performance rather than contractility is quantified (12-14). Preejection phase indices such as $\mathrm{dP} / \mathrm{dt}$ max are significantly affected by changes in preload, whereas ejection phase indices such as ejection fraction are primarily affected by afterload. To circumvent this problem, relationships have been delineated that define contractility by assessing muscle performance over a range of either preload or afterload and are independent of the other determinants. In isolated muscle preparations, the active length-tension relationship and the forcevelocity relationship are examples (15-17). Recently, the pressure-volume relationship was restudied in both the isolated right and left ventricles $(18,19)$ : by varying preload, pressure-volume loops could be constructed such that isochronal points (i.e. those that occur at the same time in the cardiac cycle) in the different cardiac cycles are related linearly. The slope of this linear relationship defines the time-varying elastance of the ventricle. At end-systole in the isolated heart, elastance is maximal (Emax) and has been shown to vary directly with changes in contractility. Modifications of this in vitro Emax have been used to measure contractility in the intact animal $(20,21)$ and human (22-27).

We have adapted the in vitro end-systolic pressure-volume relationship to the intact animal preparation in an attempt to analyze developmental changes in contractility and contractile reserve that occur in the lamb over the first month of life. We 
have also examined whether changes in preload and heart rate significantly affect this relationship and thus invalidate its use as an index of contractility. We describe results from data obtained in normal, chronically instrumented lambs.

\section{MATERIALS AND METHODS}

Surgery. Fourteen lambs of mixed western breed between 2 and 8 days of age underwent surgery. A left thoracotomy was performed in the fourth intercostal space under halothane anesthesia and supplemental $0.5 \%$ xylocaine locally. Polyvinyl catheters were placed in a hindlimb artery and vein, and in the ascending aorta and superior vena cava via the internal thoracic artery and vein, respectively, for pressure measurements and subsequent infusions. Catheters were also placed in the coronary sinus via the hemiazygous vein for myocardial metabolic studies in conjunction with the contractility studies, and directly into the left atrium for contrast injection to confirm the site of the endocardium. Within a pursestring suture and through a needle puncture, a specially designed $4 \mathrm{~F}$ microtipped manometric catheter (Millar Instruments, Houston, TX) was placed in the left ventricle through its apex. A $5.0 \mathrm{mHz}, 6.0 \mathrm{~mm}$ nonfocussed piezoelectric single crystal echo transducer (KB Aerotech, Lewiston, PA) was sutured to the left ventricular epicardium to record left ventricular wall thickness and short axis dimension at the level of the distal tip of the mitral valve. A layer of bone wax $2 \mathrm{~mm}$ thick was attached to the transducer before surgery to separate it from the epicardium in order to improve near field resolution. A precalibrated electromagnetic flow transducer was placed around the pulmonary artery. A chest tube was left in the pleural cavity. Subcutaneous electrodes used to record surface electrocardiograms were sutured in place as the skin incision was closed. All catheters and transducer cables were brought to the skin via the thoracotomy incision, and protected by a bag sutured to the animal's flank. A second group of five animals underwent the same operation at $3 \mathrm{wk}$ of age, with the additional placement of pacing wires on the left atrium.

Contractility studies. The lambs were allowed to recover for at least 3 days after surgery, a period of time found to be adequate for return of normal hemodynamic status following thoracotomy (28). Studies to measure contractility were performed with the lambs resting quietly, blindfolded, and placed erect in a sling under a heat lamp to ensure a neutral thermal environment. Continuous measurements of aortic phasic and mean pressures and heart rate were obtained and recorded on a Beckman (San Jose, CA) direct writing recorder. Cardiac output was measured with an electromagnetic flow transducer (C \& C Instruments, Culver City, CA) connected to a Statham SP2202 flowmeter (Statham Instruments, Oxnard, CA) around the pulmonary artery rather than the ascending aorta in order to avoid left ventricular obstruction during the 4th wk of study (1). The echocardiographic and left ventricular pressure signals were recorded simultaneously on an Electronics for Medicine VR12 recorder (Hayward, CA) at a paper speed of $100 \mathrm{~mm} / \mathrm{s}$ (Fig. 1). Prior to each study, the microtipped pressure transducer was calibrated externally using the aortic and left atrial catheters that were connected to Statham $\mathrm{P} 23 \mathrm{Db}$ pressure transducers. Endocardial surfaces were confirmed with an injection of $0.9 \% \mathrm{NaCl}$ solution into the left atrium while the left ventricular echo signal was recorded (Fig. 2). After an initial measurement of resting values, atropine $(0.1 \mathrm{mg} / \mathrm{kg})$ was given intravenously, followed either by a continuous infusion at $0.01 \mathrm{~g} / \mathrm{kg} / \mathrm{min}$ or repeated doses of 0.1 $\mathrm{mg} / \mathrm{kg}$ every $10 \mathrm{~min}$; this inhibited the phenylephrine-induced, baroreceptor-mediated bradycardia. The first set of measurements was made when heart rate and cardiac output stabilized. Afterward, phenylephrine was infused for about $3 \mathrm{~min}$, starting at a rate of $1 \mu \mathrm{g} / \mathrm{kg} / \mathrm{min}$ and increasing gradually to a maximum of $15 \mu \mathrm{g} / \mathrm{kg} / \mathrm{min}$. As afterload increased, we obtained left ventricular echo and pressure recordings at intervals of 5-10 torr in aortic mean pressure. When aortic mean pressure reached 115

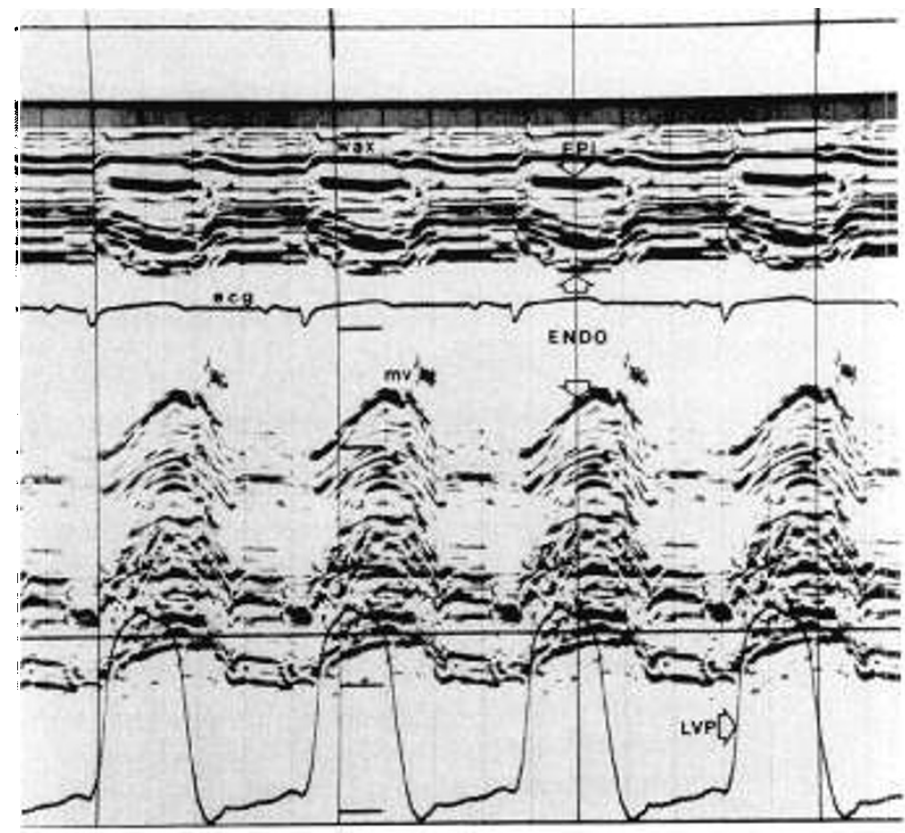

Fig. 1. Typical tracing of left ventricular echo and pressure signals. wax: wax interface on transducer. EPI, epicardium; ecg, electrocardiogram; $E N D O$, endocardium; $m v$, mitral valve; $L V P$, left ventricular pressure.

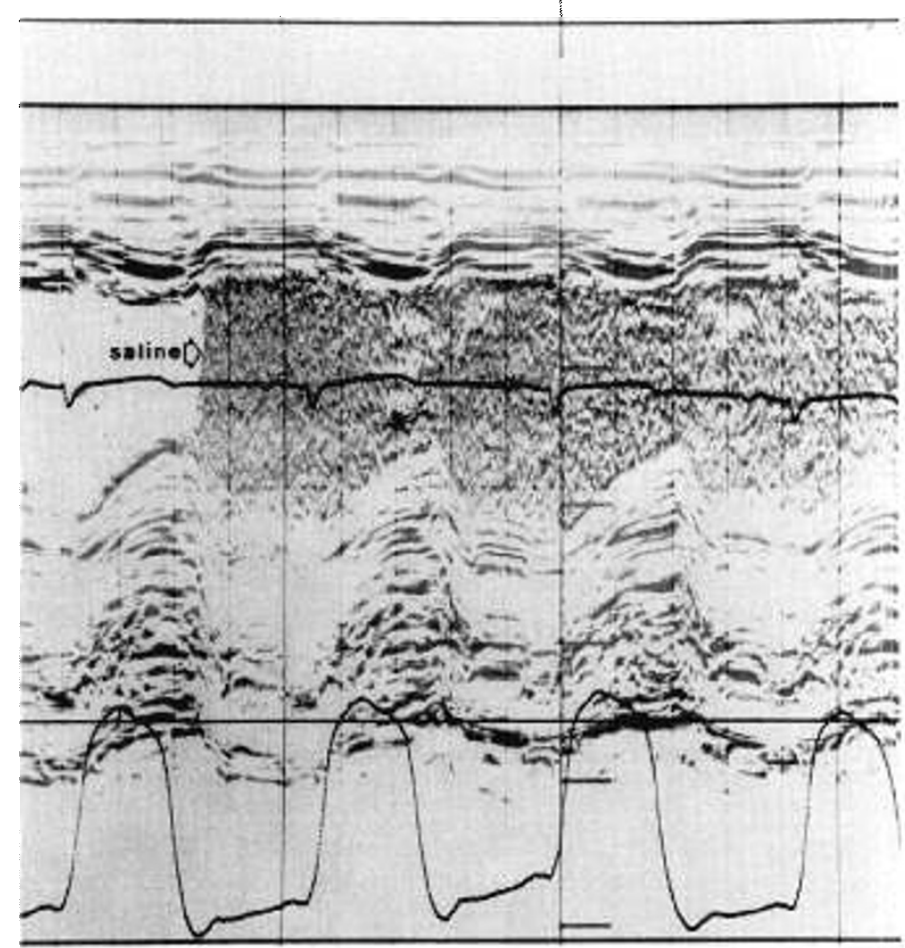

Fig. 2. Saline infusion into the left atrium to highlight endocardium.

125 torr, the phenylephrine was discontinued. In the first nine animals we then began to infuse $\mathrm{Na}$ nitroprusside, again at a rate of $1 \mu \mathrm{g} / \mathrm{kg} / \mathrm{min}$ and increasing to $5 \mu \mathrm{g} / \mathrm{kg} / \mathrm{min}$; subsequently we did not use $\mathrm{Na}$ nitroprusside but allowed the aortic mean pressure to fall normally to resting levels. Measurements were similarly recorded with every 5-10 torr drop in pressure. The study was terminated when a total of 20 measurements had been recorded, or earlier if aortic mean pressure decreased to 55 torr during the $\mathrm{Na}$ nitroprusside infusion. The animal was then allowed to 
recover to the resting hemodynamic status. One-half hour later, isoproterenol $(0.1 \mu \mathrm{g} / \mathrm{kg} / \mathrm{min})$ was infused. We chose this infusion rate to achieve maximal inotropic response; we have previously found no further increase in cardiac output at infusion rates of isoproterenol greater than $0.1 \mu \mathrm{g} / \mathrm{kg} / \mathrm{min}$ in other studies in which lambs were paced at a fixed heart rate following formalin ablation of the atrioventricular node (Teitel DF, unpublished data). After 3 min of stabilization, the complete study as outlined above was repeated. In the last four animals, we performed repeat resting studies and studies after an intravenous dose of propranolol $(1 \mathrm{mg} / \mathrm{kg})$. Resting, isoproterenol, and propranolol studies were performed at weekly intervals during the first $4 \mathrm{wk}$ of life. Two animals were not operated on until after 6 days of age and thus were studied from 2-4 wk only. There was equipment malfunction in two other animals, so they were not studied on the 4th wk.

In order to ascertain whether our index was truly one of contractility rather than global performance, we assessed whether it was affected by isolated changes in heart rate or preload. We operated on five animals at $3 \mathrm{wk}$ of age and performed the following studies $1 \mathrm{wk}$ later. To assess the possible effects of heart rate, we studied each animal on consecutive days (except one, which was studied only once) at rest, and then during left atrial pacing at a rate $30 \%$ above the resting heart rate. To assess preload effects, propranolol $(1 \mathrm{mg} / \mathrm{kg})$ was first given intravenously to abolish the $\beta$-stimulatory effects of acute volume loading. We then studied the animals prior to volume infusion and immediately after a rapid infusion of warmed normal saline $(20 \mathrm{ml} / \mathrm{kg})$. Thus resting contractility could be compared to contractility during atrial pacing, and contractility during $\beta$ adrenergic blockade could be compared to $\beta$-adrenergic blockade combined with volume loading. There should be no differences between these comparison slopes if our index of contractility is independent of changes in heart rate and preload.

Analysis. For each of the 20 measurements in each study, left ventricular wall thickness, dimension, and pressure can be measured simultaneously. From these data, wall stress and the cube of dimension can be calculated. There is little agreement about the correct in vivo point in the cardiac cycle at which to measure Emax. In the isolated heart, the time-varying elastance model defines maximal elastance as occurring at end-systole. At that time $\mathrm{dP} / \mathrm{dt}$ and $\mathrm{dV} / \mathrm{dt}$ are both zero (29). In the in vivo heart $\mathrm{dP} / \mathrm{dt}$ is zero at peak pressure whereas $\mathrm{dV} / \mathrm{dt}$ is zero at endejection. Although others have chosen end-ejection to measure Emax we chose peak pressure for two major reasons. When we compared the slopes obtained at peak pressure and end-ejection, we found that the slope at peak pressure was always greater than or equal to that found at end-ejection. Since maximal elastance is defined by the maximal slope obtained we believed that peak pressure was therefore a more appropriate time. As well, our measurements were much more reproducible at peak pressure. At end-ejection, pressure is decreasing rapidly and a minor error in timing would have a great effect on the calculation of wall stress. This is supported by our finding that the SEs of the slopes of the peak pressure measurements were always less than those of the slopes of the corresponding end-ejection measurements. After determining the time to peak pressure for the first of the 20 measurements of each study, we then chose isochronal points to make our subsequent 19 measurements during afterload alteration. This is in keeping with the method used to define the time-varying elastance model in the isolated heart $(18,19)$. The measurements were analyzed with a graphics tablet (Apple, $\mathrm{Cu}$ pertino, CA) and an Apple 2 plus microcomputer.

From the measured left ventricular pressure, wall thickness, and diameter, wall stress was calculated from the equation (30):

$$
\mathrm{WSt}=\frac{1.332 \times \mathrm{P} \times \mathrm{D}}{4 \times \mathrm{H} \times(1+\mathrm{H} / \mathrm{D})}
$$

where WSt is wall stress in $10^{3}$ dynes $/ \mathrm{cm}^{2}, \mathrm{P}$ is pressure in torr,
$\mathrm{D}$ is diameter, and $\mathrm{H}$ is wall thickness in mm. An index of left ventricular volume was made by cubing the instantaneous dimension. Since absolute slopes were not compared between animals, a more accurate measure of volume was not needed; relative changes in slopes were compared, and should not have changed by adding constants into the volume equation. Clearly, this approximation would be valid only if there were no significant changes in geometry of the heart over the $4 \mathrm{wk}$ of study. We did compare the geometry of several hearts from lambs at birth to $4 \mathrm{wk}$ of age following their arrest at end-systole during a rapid injection of $10 \%$ calcium chloride (after sedation by $\mathrm{Na}$ pentobarbital). We found no major geometric differences between animals of different ages (see "Results").

We generated approximately twenty wall stress-volume index points and wall stress-dimension points for each study. Linear regression analyses of the resultant relationships were performed. SEs of the estimate were obtained for both linear regressions, and all subsequent analyses were performed with the wall stressvolume index curves. At each week in each animal, we compared the control, isoproterenol, and propranolol slopes by using a "comparison of two slopes" test (31). Percentage change in slope was used to compare the effects of isoproterenol and age.

All the remaining statistical analyses were performed with a paired $t$ test. In all analyses differences were considered statistically significant if $p<0.05$.

\section{RESULTS}

Throughout the 4-wk study period, the lambs grew normally (Table 1). Resting heart rates and cardiac outputs were similar to those reported previously (32). There was a gradual decrease in heart rate with increasing age and a large decrease in cardiac output per kilogram.

The effects of the study protocol on heart rate and cardiac output are shown in Figures 3 and 4 . At peak phenylephrine effect the average drop in heart rate was small as a result of the vagal blockade by atropine, and statistically significant in only a few instances. In those, the absolute decrease in cycle length was never greater than $25 \mathrm{~ms}$ and did not significantly alter the position at which the measurements were taken. At the end of all studies heart rate and cardiac output were not significantly different from values at the onset, indicating that $\beta$-adrenergic tone did not change.

Figure $5 A$ and $B$ depict the points plotted from two typical studies. We performed 113 studies on 14 animals. We analyzed the data from each study by a linear regression (least squares technique): SEs of the estimate of the relationship of wall stress to volume index were less than or equal to those of wall stress to dimension; correlation coefficients were greater. We therefore used the volume index in all subsequent analyses. Correlation coefficients of the linear regression equations of wall stress versus volume index were $>0.91$ in 87 , and $>0.85$ in 101 of the 113 studies. The average SD of the calculated slope was $19 \%$ of the slope itself. Figure 6 shows the effects of isoproterenol on the slope of the wall stress-volume index line. Although there was a small average increase in slope (13\%) during $\beta$-adrenergic stimulation with isoproterenol in the $1 \mathrm{st} w \mathrm{w}$, the absolute increase was statistically significant in only one of the 10 animals studied. In the $2 \mathrm{nd}$ and $3 \mathrm{rd}$ wk of study there was a moderate increase in slope (24 and $27 \%$, respectively), with significant increases in

Table 1 . Resting values of wt, heart rate, and cardiac output (mean $\pm S D)$

\begin{tabular}{ccccc}
\hline & & Heart & \multicolumn{2}{c}{ Cardiac output } \\
\cline { 4 - 5 } Wk & Wt $(\mathrm{kg})$ & rate & $(\mathrm{ml} / \mathrm{min})$ & $(\mathrm{ml} / \mathrm{min} / \mathrm{kg})$ \\
\hline 1 & $6.0 \pm 0.8$ & $224 \pm 27$ & $1595 \pm 320$ & 265 \\
2 & $6.9 \pm 1.4$ & $215 \pm 15$ & $1705 \pm 450$ & 245 \\
3 & $8.0 \pm 1.9$ & $206 \pm 19$ & $1400 \pm 170$ & 175 \\
4 & $8.8 \pm 2.3$ & $182 \pm 20$ & $1470 \pm 330$ & 165 \\
\hline
\end{tabular}




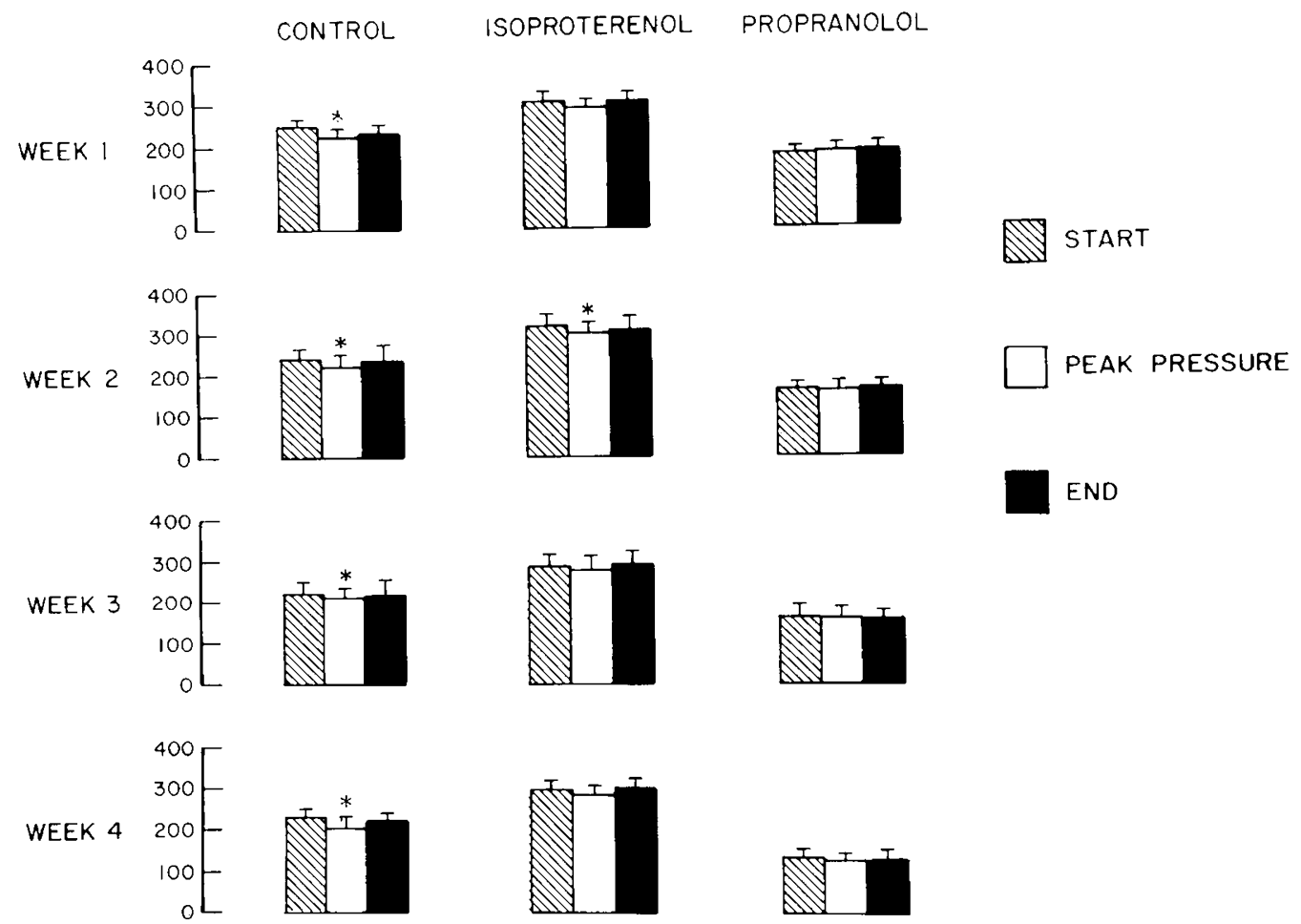

Fig. 3. Effects of the protocol on heart rate (bpm) during control, isoproterenol, and propranolol studies. Values expressed are means $+1 \mathrm{SL}$ ). * Statistically different from values at onset.

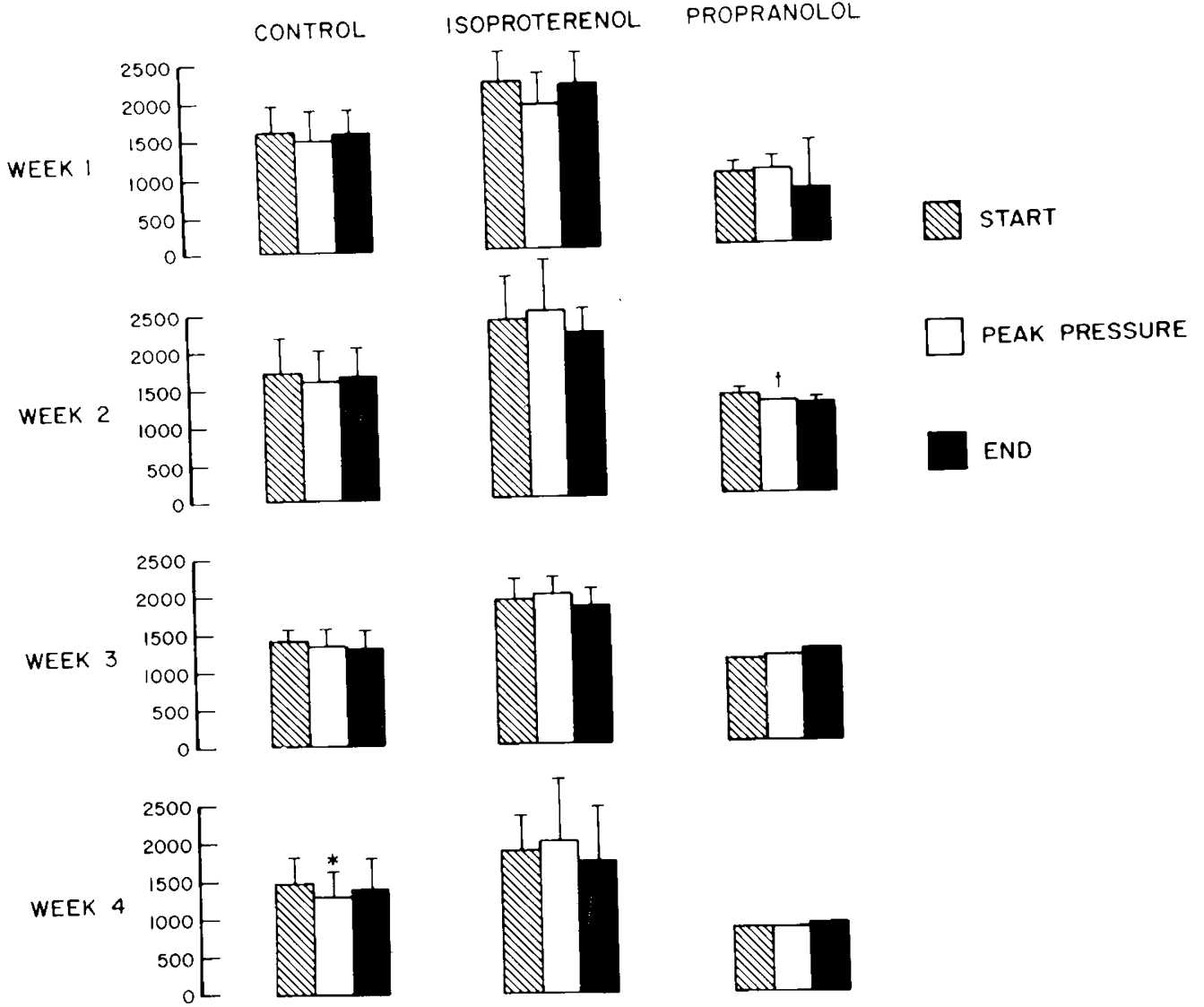

Fig. 4. Effects of the protocol on cardiac output $(\mathrm{ml} / \mathrm{min})$ during control, isoproterenol, and propranolol studies. Values expressed are means $: \pm$ $1 \mathrm{SD} . *$ Statistically different from values at onset. $†$ SD not marked when only two values were obtained. 

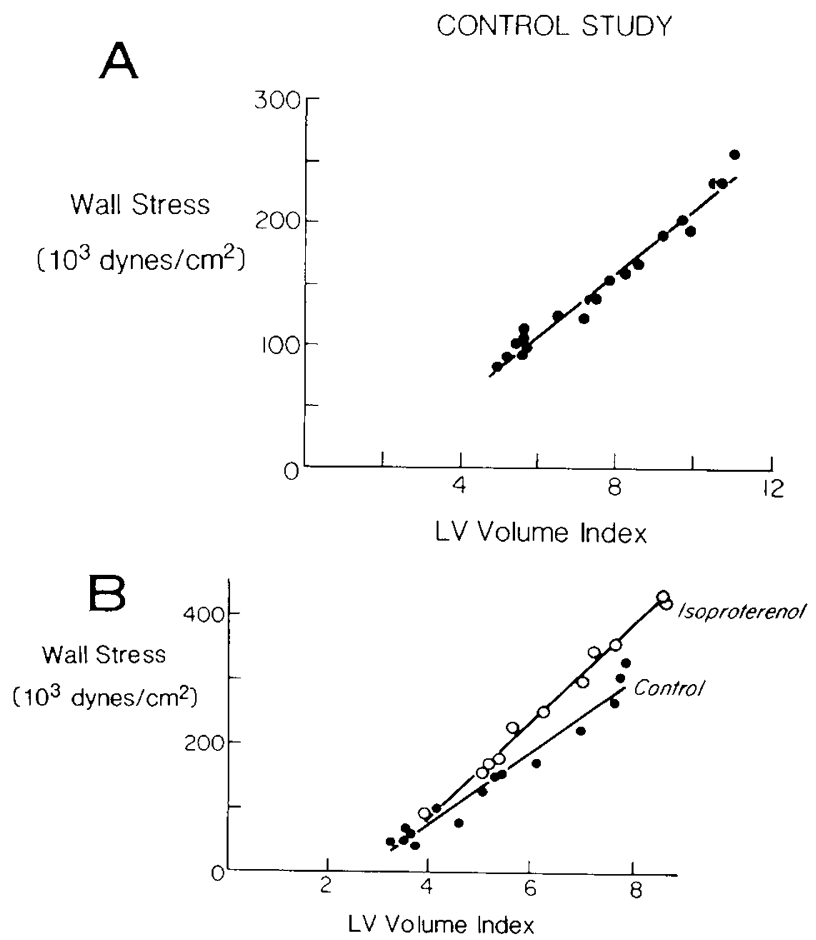

Fig. 5. Typical wall stress-left ventricular volume index lines from two animals. $A$, control study at 1 week of age; $B$, control and isoproterenol studies from another animal at $3 \mathrm{wk}$ of age showing an increase in slope with isoproterenol. LV volume index is the cube of diameter.

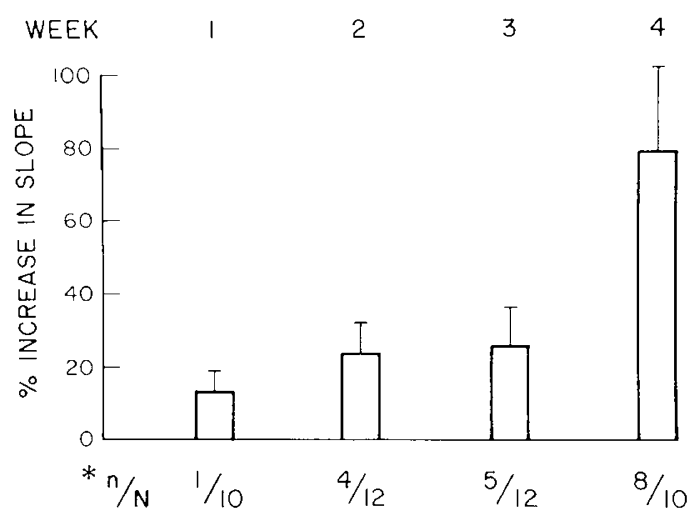

Fig. 6. The effects of isoproterenol infusion on slope at each week expressed as percentage increase from control. ${ }^{*} n / N, \mathrm{n}$ is the number of animals with statistically significant increases in slope; $N$ is the number of animals studied during that week.

four of 12 animals in the $2 \mathrm{nd} w \mathrm{w}$ and five of 12 in the $3 \mathrm{rd}$ wk. By the 4th wk the increase in slope was much greater $(79 \%)$ and in eight of 10 animals was significant. Figure $7 A$ and $B$ shows the change with age in contractility at rest and during isoproterenol infusion. When we compared the $2 \mathrm{nd}, 3 \mathrm{rd}$, and 4 th wk to the 1 st wk, we found: 1) a significant decrease in resting contractility, 2) a progressively greater number of animals with significantly less steep slopes at rest (two slope comparison test), and 3 ) that, during isoproterenol infusion, contractility was not significantly different at any age, although there were occasional individual differences. Four animals were given propranolol after resting studies. There was a significant decrease in slope with propranolol in three of the four animals at $1 \mathrm{wk}$ of age. This is in contrast with the older animals: in only one animal at $2 \mathrm{wk}$ and in none of the animals at 3 and 4 wk did propranolol cause a significant decrease in slope.

We performed nine pacing and volume studies in five animals
A

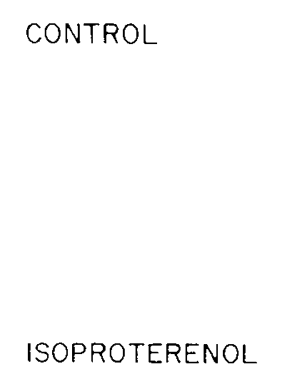

WEEK $2 \quad 3 \quad 4$

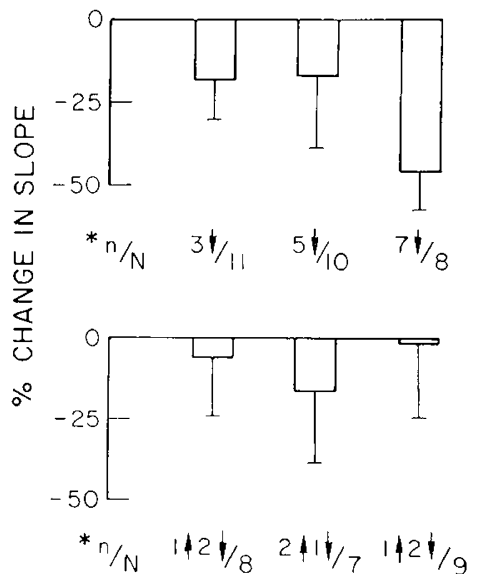

Fig. 7. Percentage change in contractility as compared to the 1st wk. $A$, resting studies; $B$, studies during isoproterenol infusion. ${ }^{*} n / N, \mathrm{n}$ is the number of animals with statistically significant increases in slope; $\mathrm{N}$ is number of animals studied during that week.

at 4 wk of age (Table 2). Atrial pacing was performed at rates averaging $36 \%$ greater than resting values, and was associated with a moderate increase in left atrial mean pressure caused by impaired ventricular filling at the rapid rates. Volume loading did not cause a reflex tachycardia because the propranolol produced $\beta$-adrenergic blockade. After the volume infusion there was a 3 -fold increase in left atrial mean pressure that persisted throughout the study period. The contractility studies showed no significant average change in slope from baseline measurements (pacing versus resting; volume loading during propranolol versus propranolol alone) during either pacing or volume infusion. In one of the pacing studies there was a statistically significant increase in slope from baseline; no increase occurred in any of the volume studies.

We compared ventricular geometry at end-systole in lambs from birth to $4 \mathrm{wk}$ of age. We measured dimensions in the long axis from apex to aortic root, and in orthogonal minor axes at the mitral annulus and midway from the annulus to the apex (representative of the dimension measured by the echo transducer). In all hearts, the ventricles were conical in shape, and the minor axes were spherical (when the papillary muscles were excluded). The ratio of midway axis to long axis was between 0.20 and 0.25 to 1 in all hearts, and there were no differences between ages.

\section{DISCUSSION}

Many indices have been devised that describe the ability of the heart to eject blood. Those indices isolated to a single measurement and taken at a discrete point in time during the cardiac cycle depend on more than one determinant of performance, and thus describe the overall pump function of the heart. However, an index of the actual muscle function, or contractility, of the heart is necessary in order to understand the effects of various physiologic processes, such as growth through maturation, hyperplasia, and hypertrophy, or the effects of various pathologic processes, such as dilatation and pathologic hypertrophy. The development of the time-varying elastance model (29) has allowed investigators to describe alterations in performance that are uniquely determined by alterations in the contractile state of the heart.

Several investigators have used modifications of the timevarying elastance model first described in the isolated heart (18, 19) as an index of myocardial contractility in the intact heart (20-27). Briefly, this model states that the maximal elastance of a ventricle in systole is dependent solely on the contractile state 
Table 2. Effects of atrial pacing and volume infusion on heart rate, left atrial pressure, and contractility (mean $\pm 1 S E M)$

\begin{tabular}{|c|c|c|c|c|c|c|c|}
\hline \multirow[b]{2}{*}{ Intervention } & \multicolumn{3}{|c|}{ Heart rate $(\mathrm{bpm})$} & \multicolumn{2}{|c|}{$\begin{array}{l}\text { Left atrial mean pressure } \\
\text { (torr) }\end{array}$} & \multirow{2}{*}{$\begin{array}{l}\text { \% Change } \\
\text { in slope }\end{array}$} & \multirow[b]{2}{*}{$\mathrm{n} / \mathrm{N}^{*}$} \\
\hline & Control & Intervention & $\%$ Increase & Control & Intervention & & \\
\hline Atrial pacing & $174 \pm 14$ & $233 \pm 17 \dagger$ & $35.6 \pm 3.7$ & $4.2 \pm 0.9$ & $12.1 \pm 1.2 \dagger$ & $+2.7 \pm 11.3$ & $1 / 9$ \\
\hline Volume loading & $147 \pm 9$ & $152 \pm 7$ & $5.7 \pm 2.5$ & $5.1 \pm 1.1$ & $15.0 \pm 1.6 \dagger$ & $-7.0 \pm 8.8$ & $0 / 9$ \\
\hline
\end{tabular}

${ }^{*} \mathrm{n}$ is the number of significant increases in slope; $\mathrm{N}$ is the number of studies performed.

+ Significant difference from control. $p<0.05$.

of its sarcomeres. Elastance is defined by the equation

$$
P(t)=E(t)[V(t)-V d]
$$

where $\mathrm{E}(\mathrm{t})$ is the time-varying elastance, $\mathrm{P}(\mathrm{t})$ and $\mathrm{V}(\mathrm{t})$ are the instantaneous pressure and volume, respectively, and $\mathrm{Vd}$ is the unstressed volume.

There are several limitations to the application of the timevarying elastance model to the intact heart. In the isolated heart, Emax occurs at end-systole, and is equal to the slope of the endsystolic pressure-volume relationship. There is, however, no discrete time in the in vivo heart to represent end-systole. Rather, end-systole incorporates the time from the onset of peak pressure to the end of ventricular ejection. Thus, some approximation of end-systole must be made to define Emax. We have discussed the reasons for our choice of peak pressure in "Material and methods." A second major limitation is the inability to measure accurately ventricular volume in the intact subject. Several techniques that can be used to extrapolate volume from dimension or area measurements include contrast or nuclear ventriculography, ultrasonic crystal or tantalum screw implantation, and two-dimensional or M-mode echocardiography. Our studies are further limited by the small size of the ventricle in the newborn lamb and the rapid growth that occurs during the 1st month of life. Thus, differences in volume and mass between repeated measures in an individual subject or single measurements between different subjects will yield different pressure-volume relationships in the absence of differences in contractility, and modifications of this relationship are necessary. Last, the baroreflex is stimulated by the large alterations in afterload that are necessary to generate multiple pressure-volume loops. Vagal discharge was blocked by atropine infusion, but adrenergic stimulation could alter contractility during the study and invalidate the measurement. In our protocol the individual studies were performed over a short period of time and there was no evidence of $\beta$-adrenergic stimulation, as heart rate and cardiac output were in all instances similar at the onset and at the end of the study. Because of the limitations described, modifications in the in vitro pressure-volume relationship are necessary in order to adapt it to the in vivo preparation, and many of the modifications are associated with a decrease in the sensitivity of the index.

We have modified the in vitro end-systolic pressure-volume relationship to assess developmental changes in contractility in the growing lamb. Specifically, we have substituted wall stress for pressure, used an index of volume based on a short axis dimension measured by M-mode echocardiography, timed our measurements to correspond to peak systolic pressure at the onset of each study, and used the slope of a linear regression analysis of the wall stress-volume index relationship as our index of contractility. Other investigators have also used wall stress in place of pressure to minimize differences secondary to hypertrophy or dilatation $(20,21,26,27)$. Sasayama et al. (21) have demonstrated the rationale for this modification. Dogs with hypertrophy secondary to aortic banding produced steeper pressure-dimension lines than did normal animals. When wall stressdimension lines were used, however, this apparent difference in measured contractility was abolished. They termed this difference "hyperfunction with normal inotropic state." Since our study follows animals as they grow over 1 month, one would expect similar changes as the left ventricular wall thickness increases. In addition, we chose an index of volume rather than dimension itself. Dimension can be used only if ventricles of similar size are compared (33). However, we assessed inotropic reserve by constructing wall stress-volume index lines at rest and during isoproterenol infusion. The associated tachycardia and vasodilation during isoproterenol infusion caused a marked decrease in ventricular volume. Had we used dimension rather than volume, the slope of the isoproterenol studies would be falsely decreased compared to control because the change in dimension would relatively underestimate the change in volume for the smaller volumes. We also compared animals at different ages as ventricular volume increased greatly. Because of the aforementioned effects of isoproterenol and age on ventricular volume, we used a more appropriate index of volume, the cube of dimension. It should be appreciated that this is a mathematical adjustment only, and does not increase the accuracy of our estimate of volume. The volume index as an estimate of volume might not be accurate if there are large changes in ventricular shape. Although we found no major geometric differences in hearts from lambs of different ages, minor configurational differences may cause the small but not significant differences in slope seen in some animals. There is a rapid increase in left ventricular volume $(34,35)$ at birth which may cause the ventricle to become more spherical transiently. In the early newborn period, our volume index would underestimate volume and overestimate slope. Because of this potential problem in comparing data from different weeks, we performed propranolol studies on the last four animals. We could then assess contractile reserve directly at each week and contrast any observed differences between weeks without comparing absolute slopes. The third modification, the timing of our measurements, has been addressed in "Materials and methods." Last, we used slope as our index of contractility. In in vitro studies of canine hearts, changes in unstressed volume occur only during regional ischemia (36). This supports the timevarying elastance model, which defines the intercept as representing unstressed, or dead, volume only, and thus it is not a factor in systolic ventricular elastance. During regional ischemia or infarction, areas of the myocardium may change their diastolic properties and thus unstressed volume would change. However, some studies in humans have shown apparent changes in unstressed dimension in normal hearts secondary to changes in contractility alone (22). We did not find consistent changes in our volume index intercept during changes in contractility and thus did not incorporate it into our index of contractility. It is important to note that we are assuming the unstressed volume of the ventricle increases similarly to absolute volume as the animals grow. If not, there would be a relative decrease in slope independent of changes in contractility, because volume would be increasing while wall stress remains relatively constant. It is extremely likely that unstressed volume does increase because there is marked hyperplasia and hypertrophy during the $1 \mathrm{st}$ month of life, and thus there is a large increase in contractile elements. However, the small trend toward a decrease in maximal slope with increasing age may have been caused by a lesser increase in unstressed volume. Future studies may be more accurate if the impedance technique, which directly estimates ventricular volume (37), can be applied to the small heart.

Even if our modifications of the in vitro pressure-volume relationship are valid, we believe it is still necessary to show that this index is independent of loading conditions and heart rate in 
the in vivo state. The control studies were carried out at heart rates and ventricular volumes different from those present during the isoproterenol and propranolol studies, and thus changes in slope may reflect changes in determinants of ventricular performance other than contractility. The range of aortic mean pressure used in all studies was similar, so afterload should not cause any differences in slope. Increasing preload by a rapid volume infusion did not change measured contractility in any of the five animals studied. Slope increased in only one of the nine studies during left atrial pacing to increase heart rate. This may have been a chance occurrence or it may have been secondary to a direct increase in contractility caused by the increase in the frequency of contraction. Similarly, there was a greater increase in heart rate during isoproterenol infusion in the older animals. It is possible that some of the increase in contractility seen in these animals may be due to heart rate effects on contractility, as defined by the force-interval relationship in isolated muscle strips (17). However, although the mechanism by which isoproterenol can increase contractility in the older animals is not fully defined, it is important that these animals do have a greater reserve to increase contractility.

We chose isoproterenol, a potent $\beta$-adrenergic stimulant, to assess contractile reserve. Other agents alter contractility through different mechanisms, and thus limitation in response to isoproterenol does not exclude a contractile response to such agents. However, cardiac glycosides have a limited effect on contractility in the newborn rabbit (38), and on myocardial performance in the newborn lamb (3). $\alpha$-Adrenergic receptors are present in high concentration in young fetal sheep but decrease rapidly throughout gestation (39) and are unlikely to be significant in the newborn. Amrinone, a positive inotropic agent in the adult which acts by an uncertain mechanism, is actually a negative inotropic agent in the newborn beagle (40). There is thus no evidence that agents that do not act via $\beta$-adrenergic stimulation have significant positive inotropic activity in the newborn, although species differences may exist and should be investigated.

Not only may geometry change with age, but also composition of the myocardium may change. If water and connective tissue content is proportionately higher in the newborn than it is in the fetus (4), both the systolic elastic and the diastolc properties of the heart would be altered. Thus comparing maximal contractile ability between ages may not be possible for reasons independent of sarcomere function. However, the presence of a reserve in contractility would not be affected by the composition of the myocardium, and we thus stress that the major difference that we found between the newborn and older lamb is not in maximal contractile ability, but in the more limited reserve that exists in the newborn. These data are congruent with studies that have employed load-dependent indices of performance. Although no other investigators have directly measured contractile reserve in the newborn, Berman and Musselman (6) measured preejection phase indices in newborn and adult sheep. The newborn showed a resting $\mathrm{dP} / \mathrm{dt}$ max that was $45 \%$ greater than the adult, and a velocity of contractile element shortening that was $59 \%$ greater at a developed pressure of 40 torr and $96 \%$ greater at a developed pressure of 10 torr. Riemenschneider et al. (7) found that the velocity of contractile element shortening increased greatly at birth to levels far above those seen in the adult. Similarly, the extrapolated maximal velocity of shortening was much higher than that seen in the adult. Moreover, during subsequent postnatal maturation, these indices declined to levels intermediate between the newborn and adult.

Our data show that there is limited increase in myocardial contractility in response to isoproterenol in the lamb during the 1 st wk of life, and that contractile reserve gradually increases in the 2 nd and $3 \mathrm{rd}$ wk and is present in most animals by the 4th wk. The two animals that showed no reserve in the 4th wk had not demonstrated any reserve previously; for some reason they apparently remained under a high degree of inotropic stimulation throughout the month. One of these animals grew poorly and weighed only $5.7 \mathrm{~kg}$ at $4 \mathrm{wk}$. Although we were not measuring $\mathrm{Hb}$ concentration when these two animals were studied, a more prolonged or severe physiologic anemia may have been present and would have caused greater adrenergic stimulation.

In order to understand why there was limited contractile reserve in the majority of the younger animals, we first compared our resting data from the different ages and later used propranolol to block $\beta$-adrenergic activity. When comparing animals, we saw heart rate and absolute cardiac output decrease progressively with age despite a large increase in weight. Also, maximal contractility apparently did not increase during isoproterenol infusion over the 1st month of life in the lambs studied. After propranolol was given, contractility decreased compared to resting levels in the first $2 \mathrm{wk}$ of life only. This absence of a negative inotropic effect of propranolol in the older lambs confirms the findings of other investigators. Klopfenstein and Rudolph (1) found a $15 \%$ decrease in cardiac output after propranolol administration in 1-wk-old lambs, but only an $8 \%$ decrease in 4-wkold lambs. Moreover, this small decrease in cardiac output was entirely abolished after pretreatment with atropine. They thus concluded that the decrease in cardiac output seen in the older lambs was secondary to the negative chronotropic effects of propranolol, and not caused by a negative inotropic effect. Similarly, Sidi et al (unpublished data) found that propranolol caused a decrease in cardiac output of only $8 \%$ in lambs about 1 month of age, which was associated with a decrease in heart rate of about $18 \%$. Thus, the high level of $\beta$-adrenergic stimulation in the neonate decreases with increasing age, and there is a coincident increase in contractile reserve.

In summary, we have adapted the in vitro end-systolic pressurevolume relationship to the chronically instrumented lamb model to assess changes in left ventricular contractility and contractile reserve. The wall stress-volume index relationship proved to be linear over a physiologic range of afterload. We applied this relationship to the lamb and found little reserve in contractility in the 1st wk of life, and a progressive increase in reserve with increasing age during the 1 st month of life. The limited reserve in the younger animals appears to be secondary to a high resting $\beta$-adrenergic state rather than to developmental differences in myocardial function.

\section{REFERENCES}

1. Klopfenstein HS, Rudolph AM 1978 Postnatal changes in the circulation and responses to volume loading in sheep. Circ Res 42:839-845

2. Romero TE, Friedman WF 1979 Limited left ventricular response to volume overload in the neonatal period: a comparative study with the adult animal. Pediatr Res 13:910-915

3. Berman W Jr, Musselman J, Shortencarrier R 1980 The physiologic effects of digoxin under steady-state drug conditions in newborn and adult sheep. Circulation 62:1165-1171

4. Friedman WF 1973 The intrinsic properties of the developing heart. In: Friedman WF, Lesch M. Sonnenblick EH (eds) Neonatal Heart Disease. Grune \& Stratton, New York, pp 21-49

5. Gilbert RD 1980 Control of fetal cardiac output during changes in blood volume. Am J Physiol 238:H80-H86

6. Berman W $J_{r}$ Musselman $J 1979$ Myocardial performance in the newborn lamb. Am J Physiol 237:H66-H70

7. Riemenschneider TA, Brenner RA, Mason DT 1981 Maturational changes in myocardial contractile state of newborn lambs. Pediatr Res 15:349-356

8. Braunwald EJ, Ross J Jr, Sonnenblick EH 1976 Mechanisms of Contraction in the Normal and Failing Heart, 2nd ed. Little Brown, Boston

9. Imperial ES, Levy MN, Zieske H Jr 1961 Outflow resistance as an independent determinant of cardiac performance. Circ Res 9:1148-1155

10. Sonnenblick EH, Downing SE 1964 Afterload as a primary determinant of ventricular performance. Am J Physiol 204:604-610

11. Strobeck JE, Sonnenblick EH 1981 Myocardial and ventricular function part 2: intact heart. Herz 6:275-287

12. Elzinga $\mathrm{G}$, Westerhof $\mathrm{N} 1979$ How to quantify pump function of the heart Circ Res 44:303-308

3. Sonnenblick EH, Strobeck JE 1977 Derived indexes of ventricular and myocardial function. N Engl J Med 296:978-982

14. Van Den Boss GC, Elzinga G, Westerhof N, Noble MIM 1973 Problems in the use of indices of myocardial contractility. Cardiovasc Res 7:834-848

15. Downing SE, Sonnenblick EH 1964 Cardiac muscle mechanics and ventricular performance: force and time parameters. Am J Physiol 207:705-715

16. Noble MIM 1978 The Frank-Starling curve. Clin Sci Molec Med 54:1-7 
17. Strobeck JE Sonnenblick EH 1981 Myocardial and ventricular function part 1: isolated muscle. Herz 6:261-274

18. Maughan WL. Shoukas AA. Sagawa K. Weisfeldt ML 1979 Instantaneous pressure-volume relationship of the canine right ventricle. Cric Res 44:309315

19. Suga H. Sagawa K 1974 Instantaneous pressure-volume relationships and their ratio in the excised. supported canine left ventricle. Circ Res 35:117-126

20. Sasayama S. Ross J Jr. Franklin D. Bloor CM. Bishop S. Dilley RB 1976 Adaptations of the left ventricle to chronic pressure overload. Circ Res 38:172-178

21. Sasayama S. Franklin D. Ross J J 1977 Hyperfunction with normal inotropic state of the hypertrophied left ventricle. Am J Physiol 232:H418-H425

22. Borow KM. Propper R. Bierman FZ, Grady S, Inati A 1982 The left ventricular end-systolic pressure-dimension relation in patients with thalassemia major. Circulation 66:980-985

23. Grossman W. Braunwald E, Mann T. McLaurin LP, Green LH 1977 Contractile state of the left ventricle in man as evaluated from the end-systolic pressure-volume relations. Circulation 56:845-852

24. Mchmel HC. Stockins B. Ruffman K. Olshausen Kv, Schuler G. Kubler W 1981 The linearity of the end-svstolic pressurc-volume relationship in man and its sensitivity for assessment of left ventricular function. Circulation $63 \cdot 1216-1227$

25. Nivatpumin T. Katz S, Scheuer J 1979 Peak left ventricular systolic pressure/ end-systolic volume ratio: a sensitive detector of left ventricular disease. Am J Cardiol 43:969-974

26. Reichek N Wilson J Sutton MStJ. Plappert TA. Goldberg S. Hirshfeld JW 1982 Noninvasive determination of left ventricular end-systolic stress: validation of the method and initial application. Circulation 65:99-108

27. Takahashi M. Sasayama S. Kawai C. Kotoura H 1980 Contractile performance of the hvpertrophied ventricle in patients with svstemic hypertension. Circulation 62:116-126

28. Sidi D. Kuipers JRG. Hevmann MA. Rudolph AM 1982 Recovery of cardiovascular function in lambs after thoracotomy. Pediatr Res 16:705-710
29. Sunagawa K, Sagawa K 1982 Models of ventricular contraction based on timevarying elastance. CRC Crit Rev Biomed Eng 7:193-228

30. Brodie BR. McLaurin LP, Grossman W 1976 Combined hemodynamicultrasonic method for studying left ventricular wall stress. Am J Cardio $37: 864-870$

31. Zar JH 1974 Biostatistical Analysis Prentice-Hall, Englewood. NJ

32. Lister G, Walter TK, Versmold HT, Dallman PR, Rudolph AM 1979 Oxygen delivery in lambs: cardiovascular and hematologic development. Am J Physiol 237:H668-H675

33. Sagawa K 1978 The ventricular pressure-volume diagram revisited. Circ Res 43:677-687

34. Kirkpatrick SE. Covell JW, Friedman WF 1973 A new tcchnique for the continuous assessment of fetal and neonatal cardiac performance. Am J Obstet Gynecol 116:963-972

35. Rudolph AM 1974 Congenital Diseases of the Heart. Year Book Medical Publishers Inc, Chicago

36. Sunagawa K, Maughan WL, Sagawa K: Effect of regional ischemia on the left ventricular end-systolic pressure-volume relationship of isolated canine hearts. Circ Res 52:170-178

37. Baan J Jong TTA Kerkhof PLM Moene RJ, Van Dijk AD Van Der Velde ET, Koops J 1981 Continuous stroke volume and cardiac output from intraventricular dimensions obtained with impedance catheter. Cardiovasc Res 15:328-334

38. Boerth RC 1975 Decreased sensitivity of newborn myocardium to the positive inotropic effects of oubain. In: Morselli PL Garattini S, Sereni F (eds) Basic and Therapeutic Aspects of Perinatal Pharmacology, Raven Press, New York, pp 191-199

39. Binah O, Legato MJ, Danilo P Jr, Rosen MR 1983 Developmental changes in the cardiac effects of amrinone in the dog. Circ Res 52:747-752

40. Cheng JB, Cornett LE, Goldfein A, Roberts JM 1980 Decreased concentration of myocardial alpha-adrenoceptors with increasing age in foetal lambs. $\mathrm{Br} \mathrm{J}$ Pharmacol 70:515-517 